\title{
ON THE NUMBER OF INVARIANT CLOSED GEODESICS
}

\author{
BY KARSTEN GROVE AND MINORU TANAKA
}

Communicated by Shing S. Chern, January 19, 1976

It is an outstanding problem in riemannian geometry whether any compact riemannian manifold of dimension $n+1>1$ has infinitely many closed geodesics. In this note we outline a proof of the following:

THEOREM. Let $M$ be a compact, 1-connected riemannian manifold and $A: M \rightarrow M$ an isometry of finite order. Then $A$ has infinitely many closed invariant geodesics if the sequence of Betti numbers for the space of maps $\sigma: \mathbf{R} \rightarrow M$ with $\sigma(t+1)=A(\sigma(t))$ is unbounded.

This is a generalization of a well-known theorem on closed geodesics $\left(A=1_{M}\right)$ by Gromoll and Meyer [2]. Observe that the assumption on the Betti numbers in our theorem is essential $\left(A=\right.$ rotation on $\left.S^{2}\right)$. Note also that the isometries of finite order are dense in the isometry group.

Outline of ProOF. Let $\Lambda(M, A)$ be the complete, riemannian Hilbert manifold of all absolutely continuous maps $\sigma: \mathbf{R} \rightarrow M$ with $\dot{\sigma}: \mathbf{R} \rightarrow T M$ locally square integrable and $\sigma(t+1)=A(\sigma(t))$ [4]. The critical points for the energy integral $E^{A}: \Lambda(M, A) \rightarrow \mathbf{R}$ correspond to $A$-invariant geodesics, and $E^{A}$ satisfies condition (C) of Palais and Smale [4]. The fixed point set of $A, \operatorname{Fix}(A)$ corresponds to the critical points with $E^{A}$-value zero, and it consists of finitely many nondegenerate critical submanifolds of $\Lambda(M, A)$. The contribution of $\operatorname{Fix}(A)$ to the homology of $\Lambda(M, A)$ is therefore at most finite dimensional.

The $\mathrm{R}$-action on $\Lambda(M, A)$ induced by translation of the parameter reduces to an $S^{\mathbf{1}}=\mathbf{R} / s \cdot \mathbf{Z}$-action, when $A$ has order $s \in \mathbf{Z}^{+}$. If $\gamma$ is a nontrivial closed $A$-invariant geodesic, it is represented by a critical point $c \in \Lambda(M, A)$ whose fundamental period is $s / m$ for some integer $m \leqslant s$. Let $s / m=s_{0} / m_{0}$, where $s_{0}$ and $m_{0}$ are relatively prime positive integers, and choose integers $n_{0}$ and $k_{0}$ such that $m_{0} n_{0}=1+s_{0} k_{0}$. Define $c^{u}: \mathbf{R} \rightarrow M$ for any $u \in \mathbf{R}$ by $c^{u}(t)=$ $c(u \cdot t)$ and put $\bar{c}=c^{1 / m_{0}}$. Then $\bar{c}$ is a critical point for $E^{A^{n_{0}}}$ with fundamental period $s_{0}$ and $\bar{c} \subset \operatorname{Fix}\left(A^{s_{0}}\right)$. For any integers $m$ and $r$ with $m s_{0}+r m_{0}$ $\neq 0, \bar{c}^{m s_{0}+r m_{0}}$ is a critical point for $E^{A^{r}}$ and $S^{1} \cdot c^{m s_{0}+m} 0, m \in \mathbf{Z}^{+}$ $\cup\{0\}$ are all the critical orbits in $\Lambda(M, A)$ "generated" by $\gamma$. In analogy to Bott [1] we find formulas for the indices and nullities of the critical orbits $S^{1} \cdot \bar{c}^{m s_{0}+r m} 0$ in $\Lambda\left(M, A^{r}\right)$ from which we derive:

AMS (MOS) subject classifications (1970). Primary 58E10; Secondary 53C20.

Key words and phrases. Isometry-invariant geodesic, equivariant degenerate Morse theory, index, nullity, characteristic invariant. 
LEMMA 1. For each integer $0 \leqslant 1<s / s_{0}$, either $\lambda\left(\bar{c}^{m s_{0}+m_{0}}, A\right)=0$ for all $m \in D_{1}:=\left\{m \in \mathbf{Z}^{+} \cup\{0\} \mid m n_{0}+k_{0} \equiv 1 \bmod s / s_{0}\right\}$ or there exist $\epsilon_{1}, a_{1} \in \mathbf{R}^{+}$such that

$$
\lambda\left(\bar{c}^{-m_{1} s_{0}+m_{0}}, A\right)-\lambda\left(\bar{c}^{-m_{2} s_{0}+m_{0}}, A\right) \geqslant\left(m_{1}-m_{2}\right) \epsilon_{1}-a_{1}
$$

for all $m_{1}, m_{2} \in D_{1}$ with $m_{1} \geqslant m_{2}$.

LEMMA 2. For each integer $0 \leqslant 1<s / s_{0}$, there exist $k_{1}, \ldots, k_{q} \in \mathbf{Z}^{+}$ and $\left\{m_{j}^{i}\right\} \subset \mathbf{Z}^{+}, j=1, \ldots, q, i>0$, such that the numbers $\left\{m_{j}^{i} k_{j}\right\}$ are mutually distinct $\left\{m_{j}^{i} k_{j}\right\}=\left\{m s_{0}+m_{0} \mid m \in D_{1}\right\}$ and

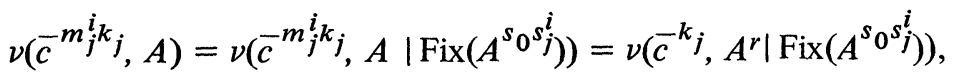

where $s_{j}^{i}$ is maximal with the properties $\left(m_{j}^{i}, s_{j}^{i}\right)=1$ and $s_{j}^{i} \mid s / s_{0}$, and where $r \in \mathrm{Z}$ satisfies $r m_{j}^{i} \equiv 1 \bmod s_{0} s_{j}^{i}$.

To each isolated orbit $S^{1} \cdot c^{m s_{0}+m_{0}}$ there is associated a local homological invariant $H\left(S^{1} \cdot \bar{c}^{m s_{0}+m_{0}}, E^{A}\right)$ which by the "generalized" Morse inequalities gives an upper bound for the contribution of $S^{1} \cdot c^{m s_{0}+m_{0}}$ to the homology of $\Lambda(M, A)[2],[3]$. The local invariant $H\left(\bar{c}^{m s_{0}+m_{0}}, E^{A}\right)$ is completely determined by the index $\lambda\left(\bar{c}^{m s_{0}+m_{0}}, A\right)$ and a characteristic invariant $H^{0}\left(c^{m s_{0}+m_{0}}, E^{A}\right)$, which in turn is determined by the degenerate part of $E^{A}$ [3].

Under the assumption that there are only finitely many closed $A$-invariant geodesics on $M$ it follows from Lemmas 1 and 2, that there are only finitely many different characteristic invariants among $\left\{H^{0}\left(c^{-m s_{0}+m_{0}}, E^{A}\right) \mid m \in \mathbf{Z}^{+}\right.$ $\cup\{0\}\}$. Furthermore, for large $k$ the number of orbits with

$$
\operatorname{dim} H_{k}\left(S^{1} \cdot \bar{c}^{m s_{0}+m_{0}}, E^{A}\right) \neq 0
$$

is uniformly bounded. Using these properties we conclude that the sequence of Betti numbers for $\Lambda(M, A)$ is bounded. Full details will appear elsewhere.

\section{REFERENCES}

1. R. Bott, On the iteration of closed geodesics and the Sturm intersection theory, Comm. Pure Appl. Math. 9 (1956), 171-206. MR 19, 859.

2. D. Gromoll and W. Meyer, Periodic geodesics on compact Riemannian manifolds, J. Differential Geometry 3 (1969), 493-510. MR 41 \#143.

3. - On differentiable functions with isolated critical points, Topology 8 (1969), 361-369. MR 39 \#7633.

4. K. Grove, Condition (C) for the energy integral on certain path spaces and applications to the theory of geodesics, J. Differential Geometry 8 (1973), 207-223. MR $49 \# 4030$.

5. - Isometry-Invariant geodesics, Topology 13 (1974), 281-292.

\section{DEPARTMENT OF MATHEMATICS, UNIVERSITY OF COPENHAGEN, COPEN- HAGEN, DENMARK}

